\title{
The application of modern Computational Fluid Dynamics techniques for increasing the efficiency and stability of an axial compressor in an industrial gas turbine
}

\author{
V. A. Sedunin ${ }^{1}$, O. V. Komarov ${ }^{1}$, V. L. Blinov ${ }^{1}$, \\ A. V. Skorokhodov ${ }^{1} \&$ A. O. Procopets ${ }^{2}$ \\ ${ }^{1}$ Turbines and Engines Department, Ural Federal University, Russia \\ ${ }^{2}$ Gazprom transgaz Yugorsk, Russia
}

\begin{abstract}
This paper contains on-site test data of an axial compressor as part of an industrial gas turbine, as well as the process of Computational Fluid Dynamics (CFD) modelling, verification, research and modernization. The research method has been verified with actual test data gathered from the real gas turbine unit (GTU) test. The computational studies showed significant potential for improving aerodynamic efficiency and the surge margin of a compressor by better matching of inlet and outer groups of stages. A special design approach was used to improve aerodynamic parameters of the inlet system and certain middle stages. Computations made for the improved compressor model showed a $3 \%$ efficiency gain and 5\% stall margin gain with possibilities for further modernization.
\end{abstract}

Keywords: axial compressor of gas turbine, CFD computations, profiling of compressor blades, verification of computational model.

\section{Introduction}

\subsection{Problem definition}

Today, the park of gas-pumping units of JSC Gazprom transgaz Yugorsk contains more than 300 units of GTK-10-4 type. During a series of retrofit actions significant changes were introduced to a design of GTU and auxiliaries. The replacement of the lamellar recuperator by a similar tubular recuperator became one of the most significant actions from the point of the reliable 
operation of the compressor. The leakages in the flow path from the compressor down to the combustion chamber were reduced to a negligible value. That caused the shift of a working point on the compressor map into an area of lower mass flow and higher pressure ratio. Owing to the wear of the compressor blading and an insufficient stall margin of the initial design, the specified actions caused unstable operation of the compressor with cases of emergency shutdowns due to surge protection system activation.

It is known that the surge of the compressor of interest happens on modes slightly exceeding the nominal corrected reference speed (from 1.00 to 1.04). For an exception of further emergency shutdowns, limitations in the loads of the compressor were introduced.

To find reasons for poor stability of the compressor and ways for its improvement special thermo-technical tests of the GTK-10-4 units were carried out, during which information on pulsations of pressure over blades at the last stages was received.

Following this, a whole blade set was measured and 3D models of the blading were designed. During computational research the corresponding conclusions and recommendations were drawn. Then the experimental set of the newly designed blading was set up on a real GTU and the corresponding tests were carried out. This article is devoted to CFD research on the axial compressor and to the experimental data of the real gas turbine with the modified geometry of the compressor.

\subsection{Background}

Historically, the development of gas turbine efficiency includes thermodynamic cycle efficiency improvement together with aerodynamic cleanness of flow path components such as turbine, axial compressor, duct, diffuser etc. Also the mass parameter is very critical for transport engines, as is the physical space for heavy duty gas turbines, for many reasons. So, together with efficiency, the loading of elements is equally as important. During the last 30 years the aerodynamic loading of the subsonic axial compressor stage has increased by $30-50 \%$ from $\frac{H_{\text {stage }}}{u_{\text {tip }}^{2}}=0.2,0.3$ to $0.4,0.5$, where $H_{\text {stage }}$ is the adiabatic work added to the flow and $u_{\text {tip }}^{2}$ is the tip circumferential rotor speed.

The higher the loading, the less the number of stages in an axial compressor. When taking into account the fact that the axial length of the compressor is 2-4 times higher than one of a turbine, reducing the number of compressor stages helps to considerably reduce the rotor length and mass of the engine.

Usually a higher loading means much greater problems with alignment of stages into one unit, especially at part load modes. This causes higher losses, the appearance of flow separation areas near meridional surfaces and requires proper flow calculations using modern robust CFD methods. Several successful results in the application of CFD methods in flow computational research in the axial compressor are presented, for example, in [3, 4].

In the mid 1970s the first attempts to implement higher aerodynamic loading were made. The gas turbine (GT) unit GTK-10-4 is one such example, where the 
tip loading coefficient exceeds 0.35 and for the first stage hub this parameter exceeds 0.6. High loading together with insufficient data about 3D flow formation theory in those times caused the misalignment of stages. Therefore the aim of the current work is the implementation of a modern compressor development experience, presented in [5-7] in existing gas turbine compressor improvement.

\section{Computational model}

3D models of the blading were designed according to the results of the measurement of a repair kit of blades. Measurements were carried out for two blades of each blade row. It should be noted that there are technological inaccuracies in the measured blades. So, by comparing the two blades of one row, the difference in profile blade angles to a radial axis or stagger angles up to 1-2 degrees were observed.

The comparison of the received models with the initial drawings was then carried out. Thus, the greatest difference is found on the leading and trailing edges: edges of real blades are up to $50 \%$ thicker than similar edges according to the drawings. The divergence in the stagger angles of separate sections reaches three degrees in profile geometry (thickness) - up to $2 \mathrm{~mm}$. This proves the fact that during its operation the constant development of the axial compressor blading was carried out repeatedly and different variations of blading take place at real units.

Historically, special attention during compressor modification was paid to the fourth stage due to frequent cases of the destruction of both blades and vanes of this stage. Also, for the inlet guide vane (IGV) the difference in an outflow angle at shroud reaches 8 degrees for various sets, which proves that a negative incidence angle in a suction side (SS) can be achieved by aiming the reduction of flow separation on the shroud.

Therefore "mixed" versions of the blading after a series of repairs is possible.

The latter significantly complicates the verification of the computational model. In the compressor, the radius of its leading edges and a stagger angle of the section have a special impact on the flow. These parameters influence efficiency and a mass flow on a nominal mode, as well as on the width of the stability range of the compressor as a whole.

A calculation was performed in Ansys CFX. The size of the element next to the wall corresponds to $\mathrm{y}^{+}<10$. The total number of cells was more than $2 \times 10^{6}$. The turbulence model was k- $\varepsilon$ standard. The interface between rows was set at the default stage. Included in the boundary conditions is the total pressure and temperature at the inlet and the static pressure at the outlet. Tip clearances are considered in mesh domains.

It is important to note that the satisfactory convergence of CFD calculation was achieved only at a small pressure ratio. Increasing the static pressure at the outlet closer to the designed value solution became unstable. First of all it can be explained by unstable behavior of the used turbulence model (k-e) operating with relatively large flow separation areas which were detected at the shroud of the 
first and last stages. However, the utilization of a similar strategy in other types of compressors gave steady convergence not only at a working point but also when $10-15 \%$ higher. The biggest deviation between calculated parameters and experimental data (up to 5-7\%) was received for values of a mass flow. This can be explained by an incorrect prediction of the blockage of an inter-blade channel with flow separation. For other parameters, differences did not exceed $1.5 \%$.

\section{Processing of the results}

In Figure 1 the distributions of Mach numbers at the root, middle and shroud section of the compressor at nominal mode are presented. From this distribution, and also from the analysis of incidence angle radial distribution at the first stages, it can be seen that the allocation of flow core down to the root section had a $0.2-0.6$ relative span. This conclusion is confirmed by observations of the real compressor after operation.

In particular, it is possible to identify the speed of a stream and existence of stagnation zones by the formation of deposits on the blade surfaces: the higher the speed, the fewer particles adhere to the surface. Proceeding from it, the flow core in the real compressor is really displaced at the root in the first stages.

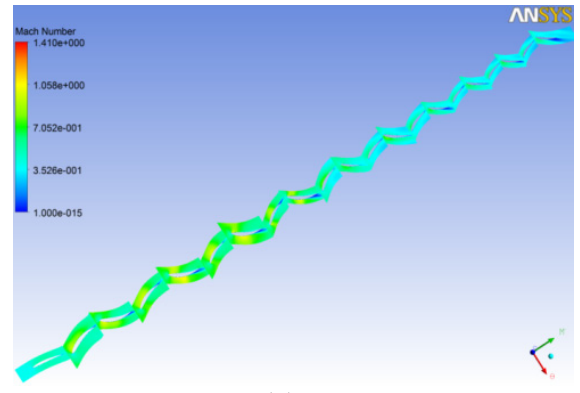

(a)

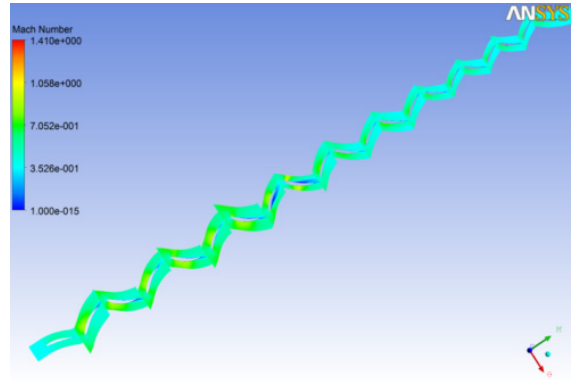

(b)

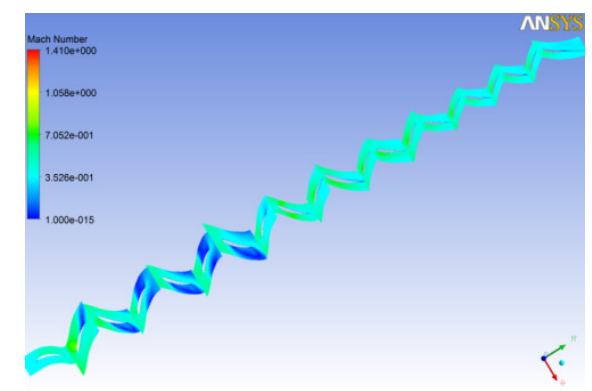

(c)

Figure 1: Distributions of Mach numbers at the root (a), middle (b) and shroud (c) section of the compressor. 
This way of flow core formation can be explained by the radial distribution law at the first stages. So these stages are designed with constant kinematic reaction distribution. For this purpose the IGV turning angle exceeds 30 degrees at the shroud (depending on a set of vanes and its producer). Thus the large turning angle is realized at the first blade row. As a result, a flow separation occurs even at the nominal mode.

In Figure 2 the radial distribution of an inlet flow angle is presented for the Stage 1 rotor blade of the axial compressor.

It should also be noted that the work added at the root section at the nominal mode is not as advanced in its designed, which leads to the lower air density in further stages. The redistribution of a flow core in the root area and also the lower air density leads to a higher incidence angle on the SS of blades and especially the vanes of the fourth stage at the $0.2-0.6$ span. In Figure 3 the radial distribution of an axial velocity at the 4 th stage outlet is presented.

In Figure 4 the radial distribution of an inlet angle is presented for the fourth stage vanes. One can see the essential incidence angle on the SS at the middle and hub regions. This even leads to a small separation on the suction side. A considerable decrease in an inlet angle at the shroud corresponds to the lower axial velocity in this area that confirms the assumptions above.

In Figure 5 the radial distribution of an outflow angle at the tenth stage is presented. One can see the angle in a flow core doesn't exceed 60 degrees, which strongly influences losses in the output diffuser. This conclusion is confirmed by the analysis of the real compressor diffuser in use: on a surface there are helicoid traces on deposits of the polluting substances, proving that the outlet from the compressor is not axial.

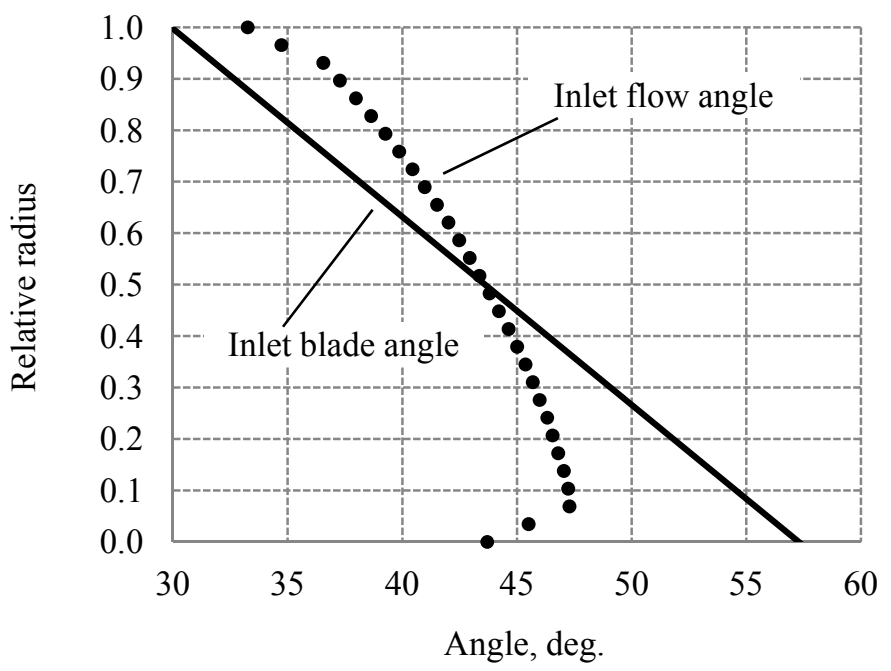

Figure 2: Radial distribution of an inlet flow angle for the Stage 1 rotor blade. 


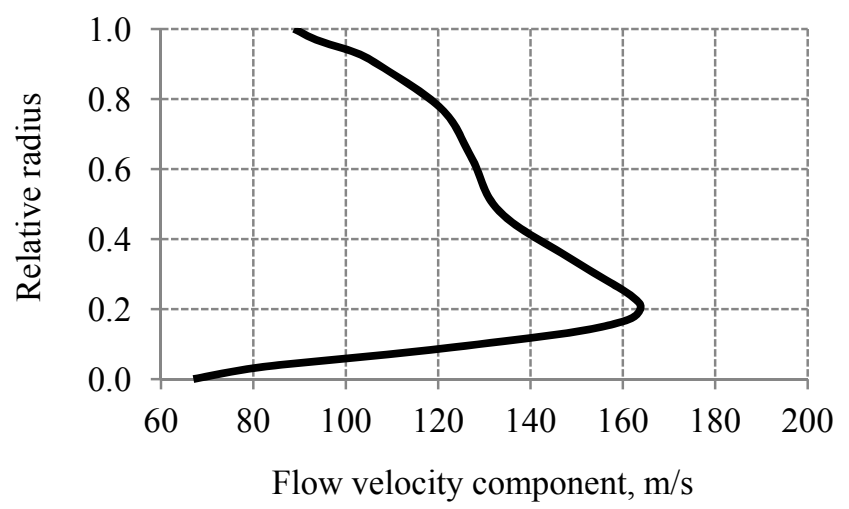

Figure 3: $\quad$ Radial distribution of an axial velocity at the 4th stage outlet.

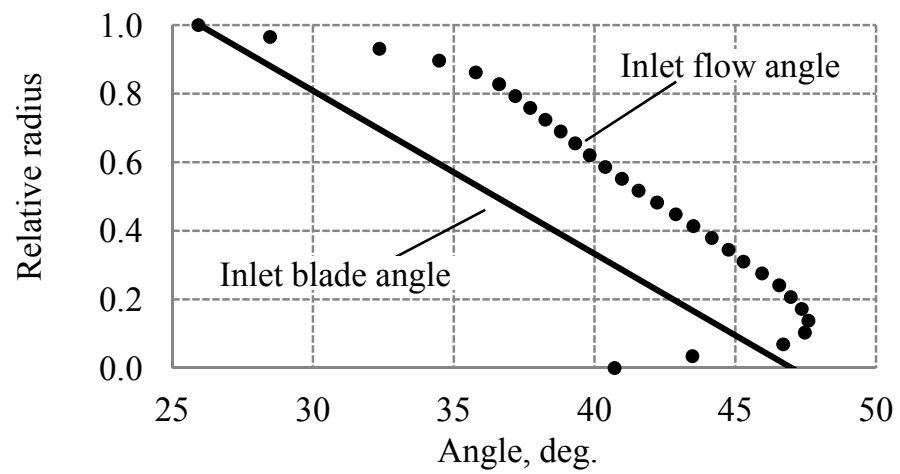

Figure 4: $\quad$ Radial distribution of an inlet angle for the 4th stage vanes.

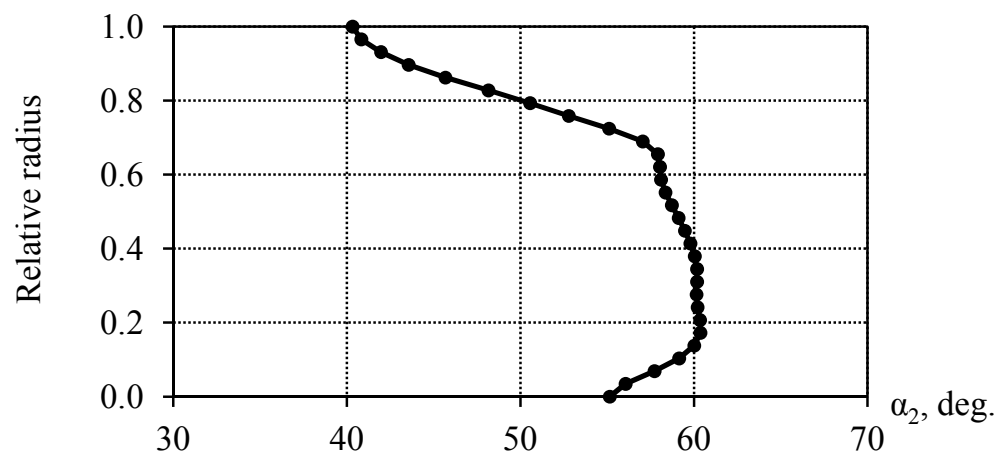

Figure 5: $\quad$ Radial distribution of an outflow compressor angle. 


\section{Variants calculations and recommendations}

By looking at the results of the conducted computational research, alternative calculations for the compressor with modified geometrics were executed. The task of minimum intervention in a design of a flow path in order to avoid the emergence of new problems with durability and dynamics of the blading was thus set. In this regard, at the first stage of calculations only vanes were exposed to change.

More than 15 various models of the compressor were studied with various changes to the geometry of IGV, blades of the first and fourth stages, and also vanes of the first, third and fourth stages. Several approaches were tested: simple re-staggering of the blade, and re-staggering of sections. The thickness of a blade and a profile form were not exposed to any change.

The greatest influence on the stability of a calculation near nominal mode and consequently on an optimality of a flow in the compressor is received at a variation of parameters of the fourth stage vanes.

Calculations were carried out at a nominal speed for different staggered angles of the fourth stage vanes: the initial blade was taken as 0 degrees, also restaggering by 2,3 and 4 degrees towards the opening were investigated further.

The greatest gain on a mass flow is observed between the constant rpm lines for the initial geometry and for a blade turned by 2 degrees. This gain is not so significant and between the line for 3 and 4 degrees the difference is comparable to the error of calculation. In this knowledge, the option of re-staggering by 3 degrees was chosen. Research was also conducted with a relative speed of 1.05 (Figures 6,7).

For the turned and initial blading, Figure 8 shows the distribution of the Mach number at the middle section of the fourth stage.

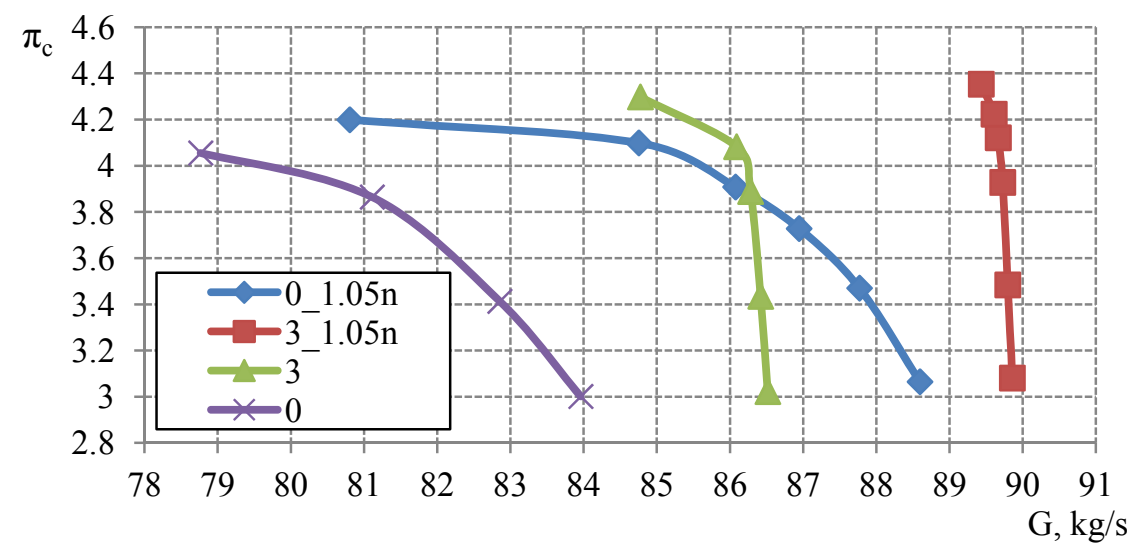

Figure 6: The mass flow vs. pressure ratio characteristic for initial and restaggered modes (radial clearance considered) (nominal rpm and 1.05 of corrected speed). 
One can see the reduction of the incidence angle on the SS in the fourth stage. Such a conclusion can be drawn due to the smaller local areas of flow acceleration on the suction side for the modified blade. It is worth noting that the design of the compressor blading with negative incidence angles at the operating mode is one of the basic standards for ensuring stability of the compressor. However in this case it is a question of the fourth stage, and, as it is known, the middle stages are less dependent on variable operating modes than the first and last stages. CFD calculations showed the incidence angle on the SS exceeding 6-8 degrees and that, certainly, is beyond rational values. In Figure 9 the radial distribution of an incidence angle at the stator 4 outlet is presented.

Due to the re-staggering of the 4 th vane, the operating conditions of the whole group of first stages was improved, in particular Figure 10 shows the distribution of a total pressure behind the first stage for the initial and modified option.

One can see that a higher radial uniformity of total pressure is achieved. From the point of view of process physics it can be explained by lower resistance at the first stages outlet, so that the working point of these stages moved to a lower pressure ratio and a higher mass flow.

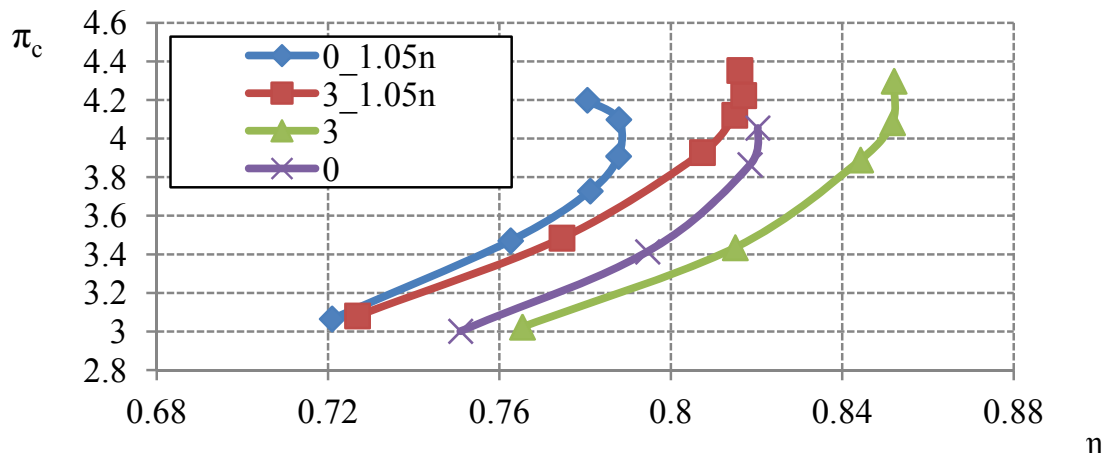

Figure 7: Efficiency-pressure ratio characteristic for initial and re-staggered modes (radial clearance considered) (nominal rpm and 1.05 of corrected speed).
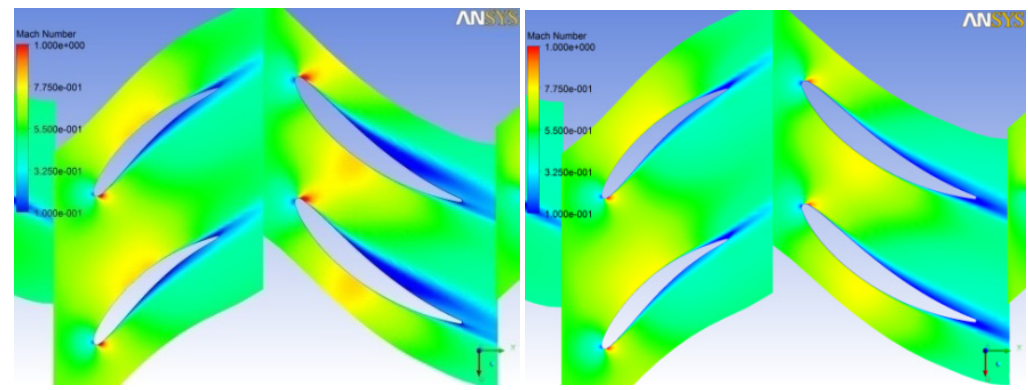

Figure 8: Distribution of Mach number at the middle section for the initial (left) and turned (right) blading. 
Computations showed that the local re-staggering of separate sections requires the essential complication of a mathematical model (like increasing the mesh size by $2-4$ times, which would raise the hardware requirements).

Thus, the most effective solution in terms of higher mass flow, higher total pressure ratio, and also a stall margin together with lowest complexity of changes proposed is the re-staggering of the fourth stage vanes by three degrees.

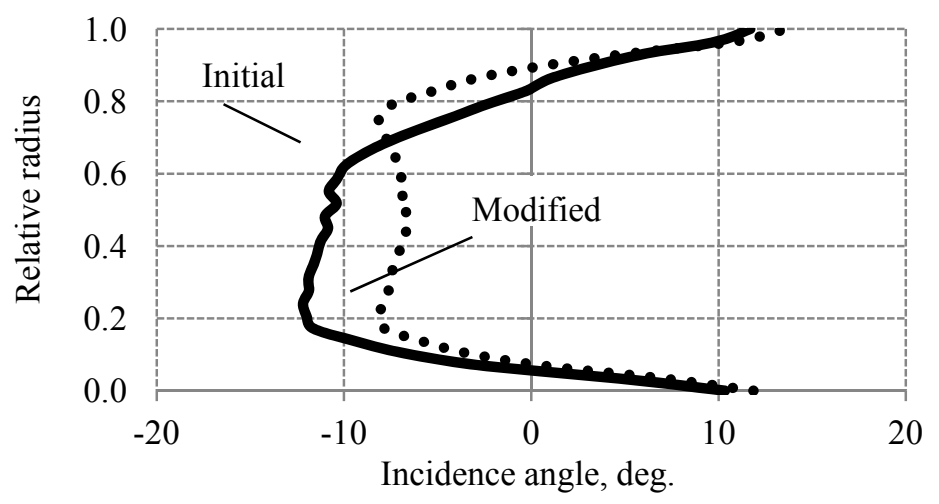

Figure 9: $\quad$ Radial distribution of an incidence angle at the stator 4 outlet.

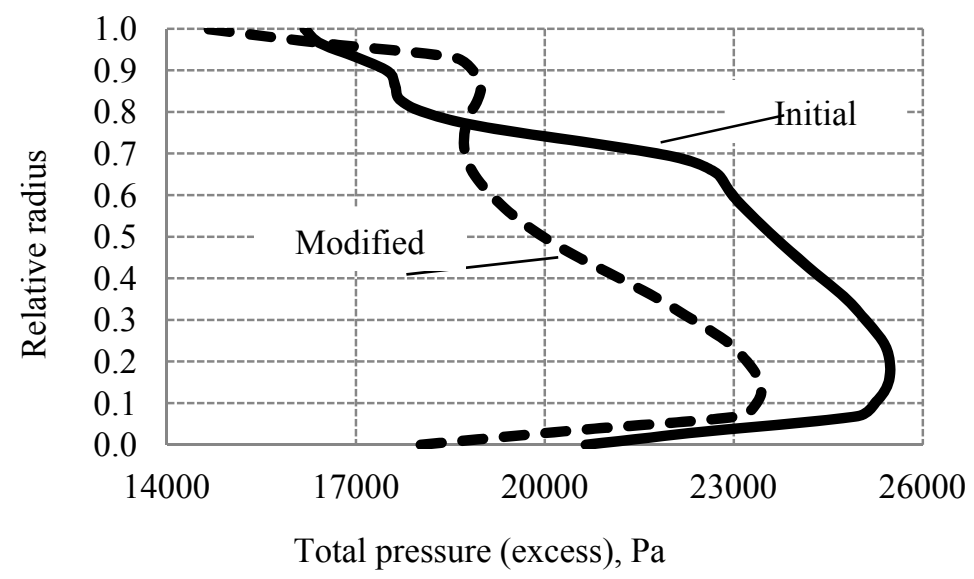

Figure 10: Distribution of a total pressure behind the first stage for the initial and modified option.

\section{Test results}

The aim of this test was to verify the computation models with the real operating unit in the widest possible range of operating modes, using the initial blading first, and also modified ones. 
During the tests, measurements of the various parameters were taken, allowing for the estimation of power, performance coefficients, and also the characteristics of the modules: the compressor, the turbine and the recuperator. Also, special measurements of the pressure over the fourth stage were performed for an assessment of the pressure ratio distribution between the inlet and outlet stages. Control of the pulsations of pressure over the tenth stage blades for an assessment of aerodynamic stability of the compressor on the nominal and increased rpm's was also carried out.

Tests were carried out on the GTK-10-4 unit of the Pangodinsky Compressor station for the initial blading, then for the modified one.

Also additional measurements, such as a full set of measurements of the natural gas compressor for the determination of its consumed power, pressure ratio, efficiency and mass flow were taken.

After the tests all the computed parameters were gathered from CFD and compared with real experimental results of the GTU. Such a comparison is given in Figure 11. Here the comparison is presented for certain operation modes gathered from test data (the ordinal number of points run horizontally). The specified range of the relative errors of values is equal to $2 \%$. In the vertical axis, the integral parameters of interest are presented.

Tests were carried out in the widest range of operating modes of the compressor as possible. GTU was slowed down to minimum load providing sustainable operation in the technology process, which means that the reduced high pressure turbine (HPT) shaft speed was around $0.82-0.85$. Then, step by step the speed was increased by up to the maximum possible load.

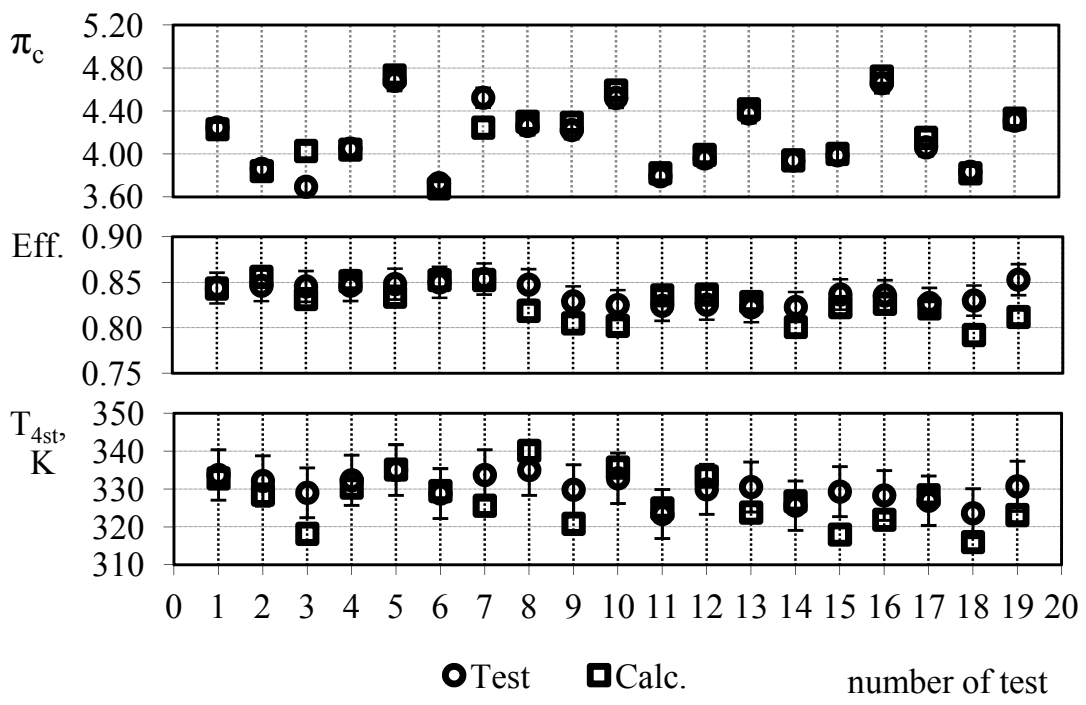

Figure 11: Comparison of the size of pressure ratio (above), polytropic efficiency (middle) and temperatures over a working blade of the fourth stage (below) according to the number of point tests. 
For the case with the initial blading the load was limited by the stability of the compressor (defined by use of the pressure pulsation sensors over last stage blades). It is known that near the nominal speed of 1.0 the last stages work closer to the stall line than the first ones.

The results of the measurements are presented in Figures 12 and 13. In Figure 12 the line of operating modes of GTU is presented. For the changed blading this line passes in the area of higher mass flow at the same rpm of a HPT shaft. It can be explained by the following: due to pressure redistribution between the first and last groups of stages, the mass flow increased (at some points up to $5 \%$ ) at a similar pressure ratio. Thus, the raised mass flow through the turbine was provided by the lower temperature of gases at the HPT entrance. On the average of similar modes by rpm, the temperature before the first turbine nozzle was 20-30 degrees Celsius lower than that for the initial blading. That led to a lower gas density in the nozzle inlet and, therefore, a higher mass flow through the turbine. This increase in mass flow was compensated by a drop in the heat within the power turbine, thus the GTU power on a similar rpm is increased by $1-2 \%$.

In Figure 13 the pressure distribution between the compressor stages is presented during the launching of the engine. The "loop" in the figure represents different moments of closing of the anti-surge valves at start-up. It can be explained by a later (higher rpm) achievement of the pressure behind the fourth stage, which causes the anti-surge valves to close. This pressure redistribution was noted throughout the whole working range.

Thus on closing of the rpm, or when exceeding nominal value, a volume mass flow at the last stages inlet is increased, which positively affects its aerodynamic stability. It is worth to noting that the proposed solution has exclusive character and is caused by the features of an initial design and should be carefully checked before implementing in the other designs.

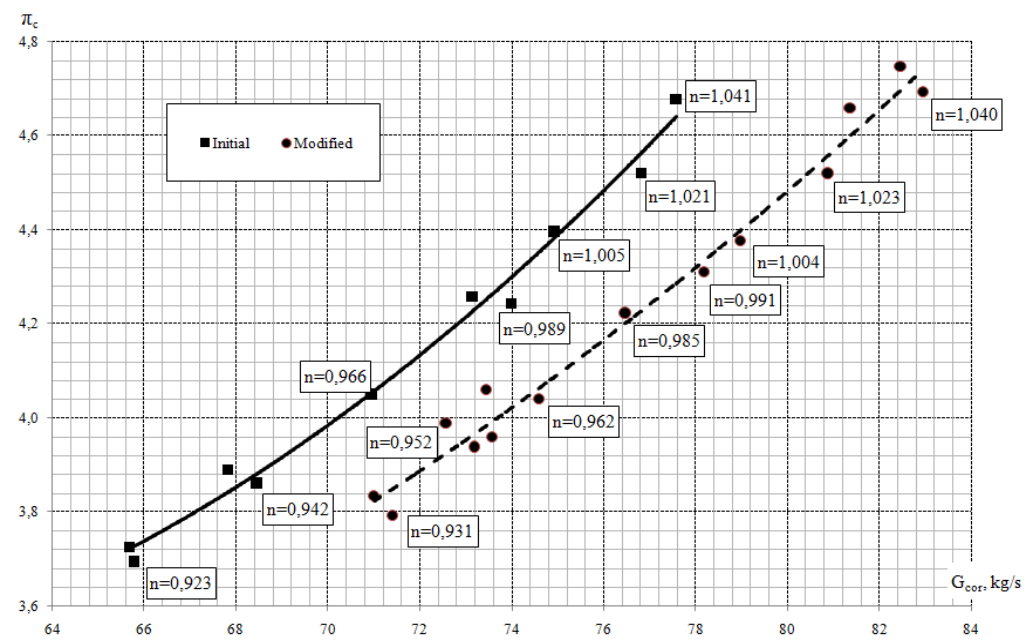

Figure 12: $\quad$ The line of operating modes of GTU. 


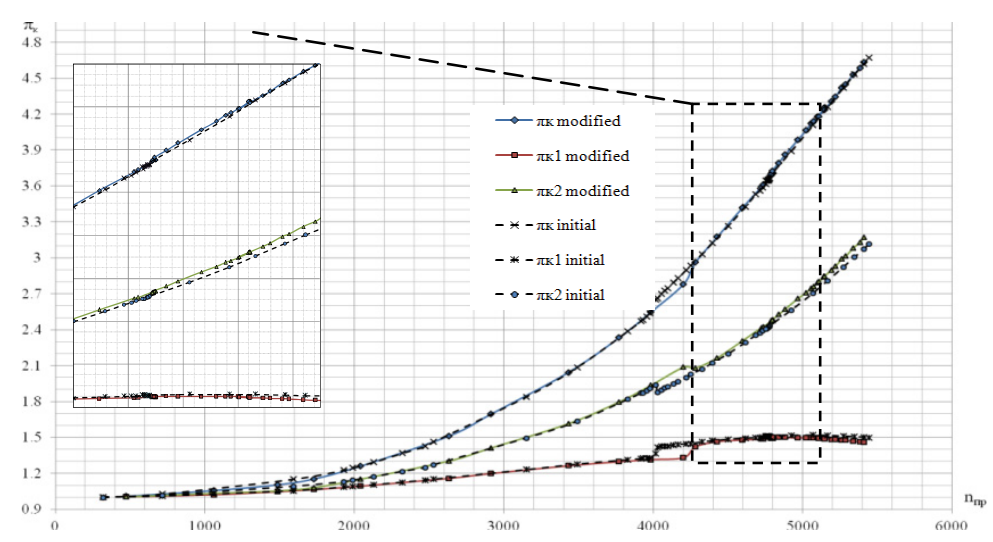

Figure 13: Change of compressor pressure ratio of the whole compressor and the inlet (1) and outlet (2) stages during start up.

\section{Conclusions}

With the computational data of the GTK-10-4 axial compressor behavior the reasons for the insufficient stall margin are detected. These are:

- The mismatch of the first and last stages of groups in the compressor can be responsible for the insufficient stall margin at the nominal and higher speed. Instability arises in a group of the last stages, which works with a volume mass flow below the optimum;

- Existence of an unstable (detachable) flow on the shroud of the first four stages causes stream redistribution by radius in such a way that the flow core is located from 0.2 to 0.6 of the relative blade height. The law of profiling of the first stages of the compressor is thus defined;

- The big swirl of the flow at the compressor outlet leads to large losses of total pressure in the outlet diffuser.

By using the results of the performed calculations, a modified profile of the fourth stage vanes is proposed. Meanwhile, the performance at variable loads shifts the characteristic curve into an area of a higher mass flow with a similar pressure ratio. Any influence on the starting characteristics is also not critical as the fourth stage is located just before the anti-surge valves.

The calculations showed that there are essential reserves in the increase of the efficiency and stability of the studied compressor.

By looking at the results of a pilot study, the efficiency of the proposed modifications is confirmed. The actions that were carried out allowed for the increase of the compressor and the GTU parameters as a whole in comparison with the initial geometry: a power gain of $2 \%$, a $5 \%$ increase of the mass flow through the compressor and an insignificant (less than 1\%) increase in total pressure ratio are achieved, together with a lower polytrophic efficiency by $1.5 \%$. 
On the basis of experimental data the specified verification of the computational model for the initial and changed geometry of the compressor was carried out. The greatest difference (up to $5 \%$ ) is received for values of a mass flow through the compressor. In other parameters the error lays in limits of the admissible range and is comparable to the accuracy of the experimental data.

The perspective directions for further improvements are the reshaping of the inlet groups of stages, providing a higher mass flow at a higher pressure ratio.

\section{References}

[1] Cumpsty, N.A., 1989, “Compressor aerodynamics”, Longman Scientific \& Technical, pp. 581.

[2] Laroisiliere, L.M. et al. Aerodynamic Design Study of Advanced Multistage Axial Compressor. NASA Technical paper TP-2002-211568, 2002.

[3] ANSYS CFX Analysis Improves Performance and Reduces Cost of 15-stage Compressor. ANSYS Solutions, Vol.7, Issue 2, 2006. pp. 29-31.

[4] Ikeguchi, T. et al. Design and Development of a 14-Stage Axial Compressor For Industrial Gas Turbine. Proceedings of ASME Turbo Expo 2012, Copenhagen, Denmark, GT2012-68524.

[5] Wellborn, Steven R., Delaney \& Robert, A., 2001, "Redesign of a 12-Stage Axial-Flow Compressor Using Multistage CFD", ASME 2001-GT-0351, presented at the ASME Turbo Expo 2001, New Orleans, LA.

[6] Sieverding, F., Casey, M., Ribi, B., \& Meyer, M., 2003, "Design of Industrial Axial Compressor Blade Sections for Optimal Range and Performance", ASME GT2003-38036, presented at the ASME Turbo Expo 2003, Atlanta, GA.

[7] Attia, M.S., Upgrade of a 16-Stage Industrial Compressor, Part II: Extension of the Analysis Method to the Design Function and Results. Proceedings of ASME Turbo Expo 2006, Barcelona, Spain, GT2006-91199. 